\title{
Tilted Magnetic Levitation Enables Measurement of the Complete Range of Densities of Materials with Low Magnetic Permeability
}

\section{Citation}

Nemiroski, Alex, Siowling Soh, Sen Wai Kwok, Hai-Dong Yu, and George M. Whitesides. 2016. "Tilted Magnetic Levitation Enables Measurement of the Complete Range of Densities of Materials with Low Magnetic Permeability." Journal of the American Chemical Society 138 (4) (February 3): 1252-1257. doi:10.1021/jacs.5b10936.

\section{Published Version}

$10.1021 /$ jacs.5b10936

\section{Permanent link}

http://nrs.harvard.edu/urn-3:HUL.InstRepos:29914187

\section{Terms of Use}

This article was downloaded from Harvard University's DASH repository, and is made available under the terms and conditions applicable to Open Access Policy Articles, as set forth at http:// nrs.harvard.edu/urn-3:HUL.InstRepos:dash.current.terms-of-use\#OAP

\section{Share Your Story}

The Harvard community has made this article openly available.

Please share how this access benefits you. Submit a story.

Accessibility 


\title{
Tilted Magnetic Levitation Enables Measurement of the Complete Range of Densities of Materials with Low Magnetic Permeability
}

\author{
Alex Nemiroski ${ }^{1 \ddagger}$, Siowling Soh $^{1 \ddagger}$, Sen-Wai Kwok ${ }^{1}$, Hai-Dong $\mathrm{Yu}^{1}$, and George M. Whitesides ${ }^{1,2,3 *}$ \\ ${ }^{1}$ Department of Chemistry and Chemical Biology, Harvard University, 12 Oxford Street, Cambridge, Massachusetts 02138, \\ United States, ${ }^{2}$ Wyss Institute for Biologically Inspired Engineering, Harvard University, 60 Oxford Street, Cambridge, Mas- \\ sachusetts 02138, United States. ${ }^{3}$ Kavli Institute for Bionano Science and Technology, Harvard University, 29 Oxford Street, \\ Massachusetts 02138, United States
}

Magnetic Levitation. Density Measurement. Quality Control. Magneto-Archimedes Levitation.

\begin{abstract}
Magnetic levitation (MagLev) of diamagnetic or weakly paramagnetic materials suspended in a paramagnetic solution in a magnetic field gradient provides a simple method to measure the density of small samples of solids or liquids. One major limitation of this method, thus far, has been an inability to measure or manipulate materials outside of a narrow range of densities $\left(0.8 \mathrm{~g} / \mathrm{cm}^{3}<\rho<2.3 \mathrm{~g} / \mathrm{cm}^{3}\right)$ that are close in density to the suspending, aqueous medium. This paper explores a simple method"tilted MagLev" - to increase the range of densities that can be levitated magnetically. Tilting the MagLev device relative to the gravitational vector enables the magnetic force to be decreased (relative to the magnetic force) along the axis of measurement. This approach enables many practical measurements over the entire range of densities observed in matter at ambient conditions-from air bubbles $(\rho \approx 0)$ to osmium and iridium $\left(\rho \approx 23 \mathrm{~g} / \mathrm{cm}^{3}\right)$. The ability to levitate, simultaneously, objects with a broad range of different densities provides an operationally simple method that may find application to forensic science (e.g., for identifying the composition of miscellaneous objects or powders), industrial manufacturing (e.g., for quality control of parts), or resource-limited settings (e.g., for identifying and separating small particles of metals and alloys).
\end{abstract}

\section{INTRODUCTION}

Magnetic levitation (MagLev) of diamagnetic or weakly paramagnetic materials suspended in a paramagnetic solution in a magnetic field gradient provides a simple method to measure density. ${ }^{1-4}$ Based the balance of gravitational and magnetic forces, this method has four important capabilities and types of applications that make it attractive for use in a variety of settings. i) MagLev offers the ability to resolve small differences in the densities of samples (e.g., pastes, gels, heterogeneous solids, small particles, crystal polymorphs $)^{1,2,4-7}$ with physical properties that make them difficult or impossible to analyze by other instruments (e.g., density gradient columns, pycnometers, oscillating-tube densometers). ${ }^{8}$ ii) MagLev can be used for complex, shape-based tasks, such as noncontact, three-dimensional self-assembly, ${ }^{9,10}$ orientation control $^{11}$, and quality control ${ }^{12}$ of a wide variety of polymeric components. iii) MagLev can be used to perform a range of important, density-based bioanalyses. ${ }^{13-17}$ iv) The simplicityof-use, portability, and low cost of MagLev make it particularly attractive for use in resource-limited settings (e.g., schools, mines, archeological sites, field operations, and laboratories in the developing countries).

Despite these advantages, one major limitation, thus far, has been an inability to measure or manipulate materials outside of a narrow range of densities $\left(0.8 \mathrm{~g} / \mathrm{cm}^{3}<\rho<2.3 \mathrm{~g} / \mathrm{cm}^{3}\right)$ close in density to the suspending, aqueous medium. To increase the range of densities that can be levitated magnetically, it is necessary to increase the force of magnetic levitation relative to gravity. Due to practical bounds to the strength of a magnetic field from a permanent magnet and the concentration of the paramagnetic salt in the medium, it has previously not been possible to increase the magnetic force enough to levitate materials with either i) low density (e.g., foams, gases) or ii) high density, in the range between $\rho \approx 2.3 \mathrm{~g} / \mathrm{cm}^{3}$ (e.g., silicon) to $\rho \approx 23 \mathrm{~g} / \mathrm{cm}^{3}$ (e.g., osmium and iridium, the two densest elements). Here, we overcome this limitation by taking a different approach: rather than increasing the magnetic trapping force, we instead decrease the gravitational force along the direction of measurement by tilting the magnetic trap relative to the gravitational vector. This method enables us to perform practical measurements over the entire range of densities observed in matter at ambient conditions (i.e., from air bubbles to osmium and iridium).

Figure 1a shows a schematic of the MagLev device that we have used in most of our previous work. This configuration consists of two, anti-aligned magnets (that is, parallel, with like-poles facing each other) and a nonmagnetic container filled with paramagnetic solution (typically aqueous $\mathrm{MnCl}_{2}$ or $\mathrm{GdCl}_{3}$ ) with density $\rho_{m}$ placed between the two magnets. A non-magnetic sample, with density $\rho_{s}$, levitates in the paramagnetic solution due to the balance of two forces: the gravitational (i.e., buoyant) force $F_{g}$ caused by a difference in density between the solution and the sample and the magnetic force $F_{m}$. The magnetic force originates from the difference in the magnetic energy density between the paramagnetic solution and the diamagnetic (or weakly paramagnetic) object: it is more energetically favorable for a volume of paramagnetic solution to be in a region of high magnetic field (i.e., close to 
the faces of the magnets) than for the same volume of a nonmagnetic object. The paramagnetic medium, therefore, displaces the object away from the magnets and towards the midpoint between the faces of the magnets. The gravitational force, however, opposes the magnetic force and acts to pull the sample down from the center (if $\rho_{s}>\rho_{m}$ ) or to displace the sample up from the center (if $\rho_{s}<\rho_{m}$ ); the objects levitates at the position where $F_{\mathrm{m}}=F_{\mathrm{g}}$. Importantly, if the sample is sufficiently dense (e.g., $F_{\mathrm{g}} \gg F_{\mathrm{m}}$ ) that it rests at the bottom of the device and does not levitate, standard MagLev is not applicable without a change in experimental procedure.

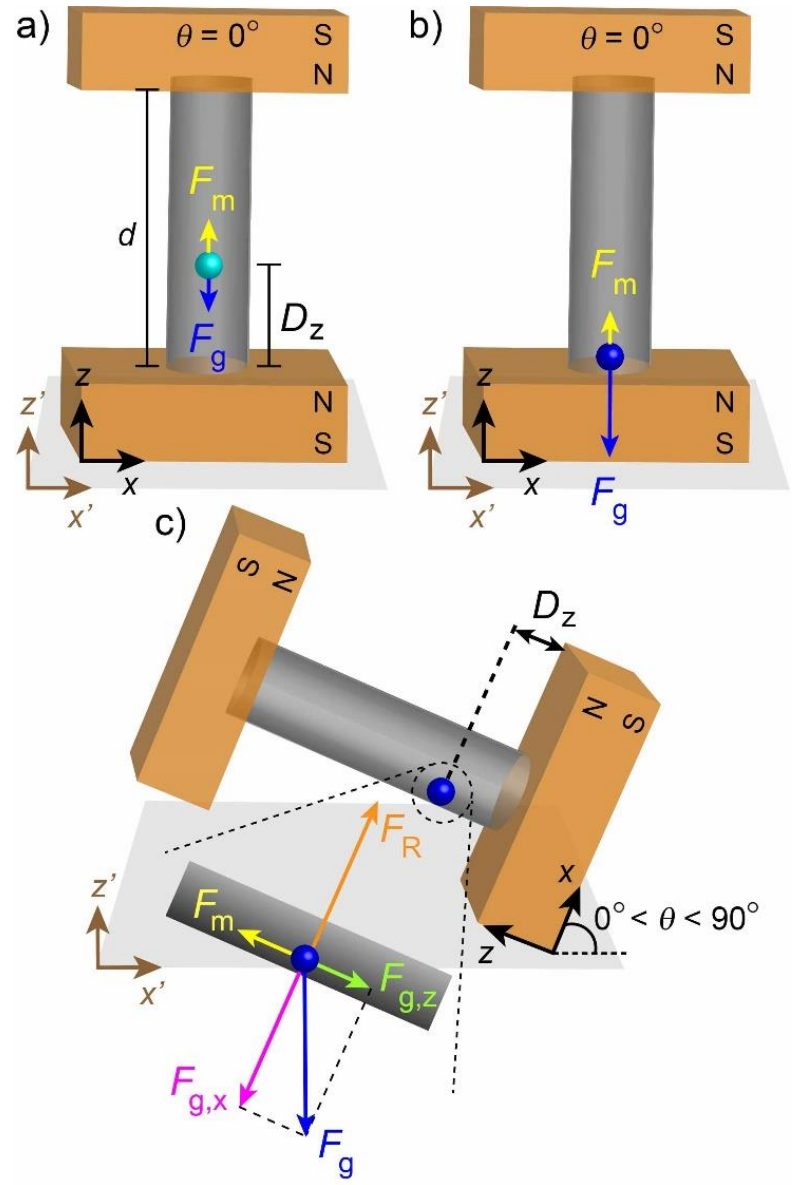

Figure 1. The magnetic levitation (MagLev) device used to measure densities of non-magnetic objects in a paramagnetic solution. (a) Schematic of an object levitating in a MagLev device. Objects levitate in the device due to the balance of the gravitational force, $F_{\mathrm{g}}$, and the magnetic force, $F_{\mathrm{m}}$. By measuring the distance $D_{\mathrm{z}}$ between the object and the surface of the bottom magnet, the density of the object can be calculated. (b) Schematic showing an object that is too dense to be measured by this configuration. (c) Schematic of a MagLev device tilted to an angle $\theta$ relative to the gravitational axis. The $x$ and $z$ axes are defined as fixed with the device and rotate with the angle $\theta$; the $x^{\prime}$ - and $z^{\prime}$ axes are fixed to the laboratory frame of reference, and do not rotate. Tilting the device provides a way to tune the components of the gravitational force $\left(F_{\mathrm{g}, \mathrm{x}}, F_{\mathrm{g}, \mathrm{Z}}\right)$ relative to the magnetic field and the z-axis (the axis of measurement) and bring the object into the range of the device. The same method can be applied to an object that is less dense than the solution; in this case, the object floats to the top of the container rather than sinking to the bottom. $F_{R}$ represents the normal force acting from the surface of the container onto the object.
Figure 1 also defines our choice of coordinates for the MagLev frame of reference ( $\mathrm{x}-, \mathrm{y}-$, and $\mathrm{z}$-axes; fixed to the device) and laboratory frame of reference ( $x^{\prime}-, y^{\prime}-$, and $z^{\prime}-$ axes; fixed to the laboratory). For many magnetic configurations, the z-component of the magnetic force $F_{m}$ can be closely approximated by a linear relationship between the height of levitation $D_{z}$ (the distance between the object and the surface of the bottom magnet) and $\rho_{s}$. Equation 1 (described by Mirica et. al. $)^{2}$ quantifies this relationship in terms of the different magnetic susceptibilities between the sample and medium $\Delta \chi=\chi_{s}-\chi_{m}$ (unitless), the magnetic strength at the surface of the magnets $B_{0}(\mathrm{~T})$, the magnetic permeability of free space $\mu_{0}$ $\left(4 \pi \times 10^{-7} \mathrm{~N} / \mathrm{A}^{2}\right)$, and the distance of separation between the two magnets $d(\mathrm{~m})$.

$$
\mathbf{F}_{\mathbf{m}}=\frac{4 \Delta \chi B_{0}{ }^{2} V}{\mu_{0} d^{2}}\left(\frac{d}{2}-D_{z}\right) \hat{\mathbf{z}}
$$

Equation 2 gives the gravitational force $\mathbf{F}_{\mathrm{g}}$, where $\Delta \rho=\rho_{s}$ $\rho_{m}\left(\mathrm{~kg} / \mathrm{m}^{3}\right)$ is difference in density between the sample and medium, $V\left(\mathrm{~m}^{3}\right)$ is volume, and $g\left(-9.8 \mathrm{~m} / \mathrm{s}^{2}\right)$ is the constant of gravitational acceleration.

$$
\mathbf{F}_{\mathbf{g}}=\Delta \rho V g \hat{\mathbf{z}}^{\prime}
$$

Equation 3 describes the linear relationship between $\Delta \rho$ and $D_{z}$ in the standard configuration (where the $\mathrm{z}$ - and z'-axes are parallel, as shown in Figures 1a,b) at equilibrium $\left(\mathbf{F}_{\mathbf{m}}=\mathbf{F}_{\mathbf{g}}\right)$.

$$
\Delta \rho=\frac{4 \Delta \chi B_{0}^{2}}{\mu_{0} g d^{2}}\left(D_{z}-\frac{d}{2}\right)
$$

For this configuration, Equation 4 describes the range of densities $\Delta \rho_{\text {range }}$ that can levitate without contact within the MagLev device.

$$
\Delta \rho_{\text {range }} \approx \Delta \rho(d)-\Delta \rho(0)=\frac{4 \Delta \chi B_{0}{ }^{2}}{\mu_{0} g d}
$$

In this paper, we show how to modify these relationships to decrease the effective $g$, and therefore, to increase $\Delta \rho_{\text {range }}$, by rotating the $\mathrm{z}$-axis of the MagLev device relative to the gravitational vector. This approach enables us to tune the strength of the linear relationship between $\Delta \rho$ and $D_{z}$, independent of all other parameters of the system.

\section{EXPERIMENTAL}

Expanding the Range of Levitated Densities by Rotation Relative to Gravity. We define $\theta$ as the angle of tilt about the $\mathrm{y}$-axis $\left(\hat{\mathbf{y}}|| \hat{\mathbf{y}}^{\prime}\right)$, i.e., the angle between the $z$ - and $z$ '- axes. Equations 5 and 6 describe how, for an arbitraty tilt angle $\theta$, the gravitational force can be decomposed into two components within the MagLev frame of reference: $F_{g, x}$ along the x-axis and $F_{g, z}$ along the z-axis.

$$
\begin{gathered}
F_{g, x}=\mathbf{F}_{\mathbf{g}} \cdot \hat{\mathrm{x}}=\Delta \rho V g \sin \theta \\
F_{g, z}=\mathbf{F}_{\mathbf{g}} \cdot \hat{\mathrm{z}}=\Delta \rho V g \cos \theta
\end{gathered}
$$

A dense (light) object resting at the bottom (top) surface of the container (e.g., as depicted in Figure 1c) will experience a normal force $F_{R}=F_{g, x}$ exerted by the wall of the container. The component of the bouyant force acting along $\mathrm{z}$-axis (the axis of measurement) $F_{g, z}$, and therefore, the amount of bouyant displacement from the center of the device, will decrease by a factor of $\cos \theta$. Equations 7-8 describe how changing $\theta$ effects the relationship between $\Delta \rho$ and $D_{z}$, as well as $\Delta \rho_{\text {range }}$, at equilibrium (i.e., when $F_{m}=F_{g, z}$ ). 


$$
\begin{aligned}
& \Delta \rho=\frac{\Delta \chi 4 B_{0}{ }^{2}}{\mu_{0} g \cos \theta d^{2}}\left(D_{z}-\frac{d}{2}\right) \\
& \Delta \rho_{\text {range }} \approx \frac{\Delta \chi 4 B_{0}{ }^{2}}{\mu_{0} g \cos \theta d}
\end{aligned}
$$

Approaches to Reduce the Effects of Friction on Equilibrium Levitation Height. Any object in contact with the surface of the container will experience a force of static and dynamic friction from the wall of the container. In practice, we found that, especially for non-spherical objects (i.e., those than cannot roll easily), this force is often large enough to impede movement of the object along the $\mathrm{z}$-axis enough to prevent it from reaching the equilibrium position, where $F_{m}=$ $F_{g, z}$

To overcome this challenge, we developed two different approaches that helped reduce or even eliminate the effect of friction. i) For spherical objects, we agitated the container by gentle, manual tapping or rolling of the container back and forth around the z-axis. ii) For non-spherical, millimeter-sized objects, we increased the viscosity of the medium such that, after tilting the device to an angle $\theta$, rotation of the container by $180^{\circ}$ about the z-axis would "pick up" the samples and then allow them to drop back to the bottom surface. This approach enabled the objects to approach their equilibrium position without having to overcome the static friction from physical contact with the wall of the container. Using these procedures, we demonstrate the measurement of an extremely broad variety of densities, from an air bubble $\sim 0.0 \mathrm{~g} / \mathrm{cm}^{3}$ to osmium and iridium (to $23 \mathrm{~g} / \mathrm{cm}^{3}$ ), as well as powders (including those with particle sizes $<100 \mu \mathrm{m}$ ) and multiple objects simultaneously.

Materials, Configuration, and Parameters. A cylindrical glass vial or a rectangular, quartz cuvette served as the container for the sample and paramagnetic medium: $3.00 \mathrm{M}$ $\mathrm{MnCl}_{2}$, for which we calculated a value of $\chi_{m}=5.48 \times 10^{-4}$ following the procedure described in Mirica et. al., ${ }^{2}$ and measured $\rho_{\text {sol }}=1.292 \mathrm{~g} / \mathrm{cm}^{3}$ with a portable density meter (DMA35, Anton Paar). We used three different sets of N40 magnets with $B_{0} \sim 0.4 \mathrm{~T}$ with $\mathrm{LxWxH}=4$ "x4"x1" (kjmagnetics.com) and assembled a custom jig to fix anti-aligned configuration at $d=$ $4.5 \mathrm{~cm}$, for all experiments.

Procedure for Levitating Spherical Samples. To characterize a spherical sample, we tilted the MagLev device to a random angle, and allowed the spherical object to assume its equilibrium position in the container. If the angle of tilt, $\theta$, was too small, the spherical object would roll and settle at the end of the container close to the bottom magnet (after agitation). If a sphere remained at the same position, regardless of manual agitation, we assumed it had reached its equilibrium position. Once this condition was met, we measured $\theta$ and $D_{z}$, and used Equation 7 to calculate the density of the object.

Procedure for Levitating Non-Spherical Samples. To characterize non-spherical samples, we prepared a highly viscous medium composed of aqueous dextran ( $35 \%$ by weight) in $3.00 \mathrm{M} \mathrm{MnCl}_{2}$; for this solution, we measured $\rho_{m}=1.385$ $\mathrm{g} / \mathrm{cm}^{3}$. This highly viscous liquid (viscosity $\sim 10 \mathrm{~Pa} \cdot \mathrm{s},{ }^{18}$ or approximately that of honey) enabled us to use viscous drag to "pick up" the sample and rotate it with the container, about the z-axis. Figure 2 shows a schematic of this procedure: after rotating the container by $180^{\circ}$ and then stopping, the sample fell gradually back to the bottom surface of the container.
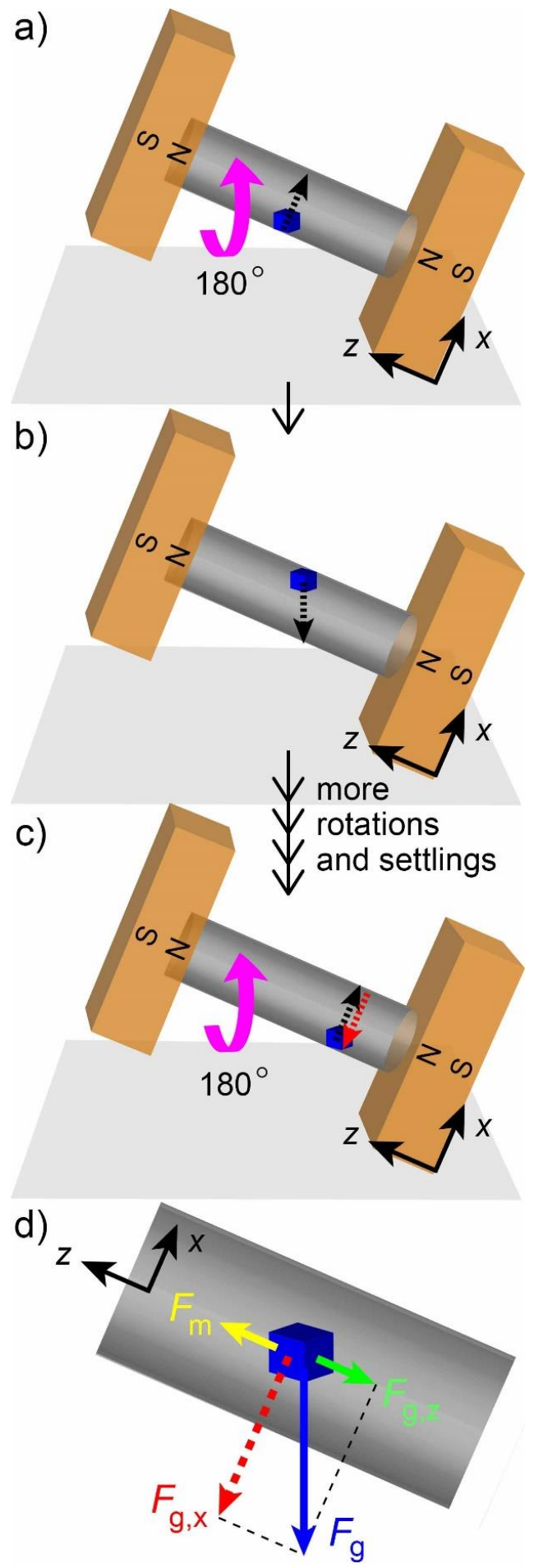

Figure 2. Schematics showing the procedure by which we measured densities of dense, non-spherical, millimeter-sized objects. When the container was rotated $180^{\circ}$ about the $z$-axis (a), the object rotated together with the container (due to the force of viscous drag from the solution) and moved to the top (b). After rotation, the object sank toward the bottom surface of the container. With more rotations, the object moved to the equilibrium position, where $F_{\mathrm{m}}=F_{g, z}$. c) Once it reached an equilibrium position, the object would no longer change its z-position after repetition of steps (a)-(b). d) A schematic showing the forces acting on the object while falling through the medium. 
We repeated this procedure several tens of times (depending on the size, density, and shape of the sample) until the bottom position along the $\mathrm{z}$-axis would no longer change from turn to turn. In this case, we assumed that the object had reached its equilibrium position along the $\mathrm{z}$-axis. Once this condition was met, we measured $\theta$ and $D_{z}$, and used Equation 7 to calculate the density of the object. Without a viscous liquid (i.e., a paramagnetic solution without dextran), we could not "pick up" the samples; they continued to stay at the bottom regardless of the rate of rotation about the z-axis.

Procedure for Levitating Powders. To characterize powders, we also used 3.00- $\mathrm{M} \mathrm{MnCl}_{2}$ and chose the viscosity such that the powders didn't settle too quickly, but settled fast enough to reach equilibrium within minutes. For powders of glass and aluminum, we used a solution with no added dextran. For tin and copper powders, we added 10\% (by weight) dextran (viscosity $\sim 0.2 \mathrm{~Pa} \cdot \mathrm{s})^{18}$. For gold powder, we added $35 \%$ dextran (by weight).

As in the case of non-spherical particles, we facilitated equilibriation by agitating the container. Although the powders tended to disperse throughout the container during the initial rotations, after more rotations $(\sim 20)$, the particles gradually converged into a narrow band (see SI for experimental images of gold powder in the SI). Alternatively, we found that decreasing the angle of periodic rotation to $\sim 90^{\circ}$ (instead of $180^{\circ}$ ) around the $\mathrm{z}$-axis allowed the particles to remain settled at all times while sliding along the surface of the container. This approach enabled us to prevent dispersion of the particles throughout the solution and attain equilibrium after only several rotations.

We include further details of all experimental procedures, materials, parameters (e.g., magnetic susceptibilities of samples), and methods in the Supporting Information (SI).

\section{RESULTS AND DISCUSSION}

Measuring Densities of Spherical Samples. Figure 3a demonstrates how, at sufficiently large $\theta$, a spherical sample would roll and come to rest at an equilibrium position $D_{z}$ separated from the bottom magnet. For each sample, we repeated the experiments using different values of $\theta$ (and as a result, different values of $D_{z}$ ). Table 1 lists the measured densities of different spherical objects, each averaged over all of the different angles of measurement. These values are in close agreement with those reported by the manufacturer or from other sources (SI).

Measuring Densities of Non-Spherical Samples. Figure $3 \mathrm{~b}$ shows measurements performed on four non-sphereical samples of different materials, using a highly viscous medium. Table 2 shows the measured densities of a broad range of objects spanning air to osmium. In each case, to reach equilibrium, we agitated the sample by periodic rotation of the container around the z-axis (Figure 2). By this approach, we are able to measure the densities of the two densest, common, naturally-occurring elements known-osmium and iridium $\left(\rho_{\mathrm{s}}\right.$ $\sim 23 \mathrm{~g} / \mathrm{cm}^{3}$ ). We also demonstrated the capability of the device to characterize substances with low density $\left(<0.8 \mathrm{~g} / \mathrm{cm}^{3}\right)$ by measuring the density of an air bubble within the container.

We observed that the repeatability of this method (as applied to non-spherical objects) was strongly dependent on the geometry of the samples. For example, the flat piece of diamond (shown in the top left image of Figure 3b), yielded a particuarly high variability due to the variety of positions it tumbled into when it came to rest on the bottom surface. To overcome this variability, we rotated the container continuously and rapidly enough to prohibit the sample from settling at the bottom of the container. We present a more detailed discussion regarding the physical origin of this behavior and the method for reducing this variation in the SI.

Although we have focused primarily on solid phase samples in this work, it may also be possible to measure liquid or vapor-phase samples by tilted MagLev as well. (We have, for example, demonstrated here the measurement of an air bubble without issue.) There is, however, a potential challenge that would need to be overcome: the samples may stick to the walls of the container due to wetting on a rough surface or contact angle hysteresis, therby preventing them from reaching an equilibrium position. Although agitation may help, in principle, adding an appropriate surfactant may aleviate the issue entirely by enabling the sample to "roll" along the wall on a thin film of the medium trapped between the sample and the wall.

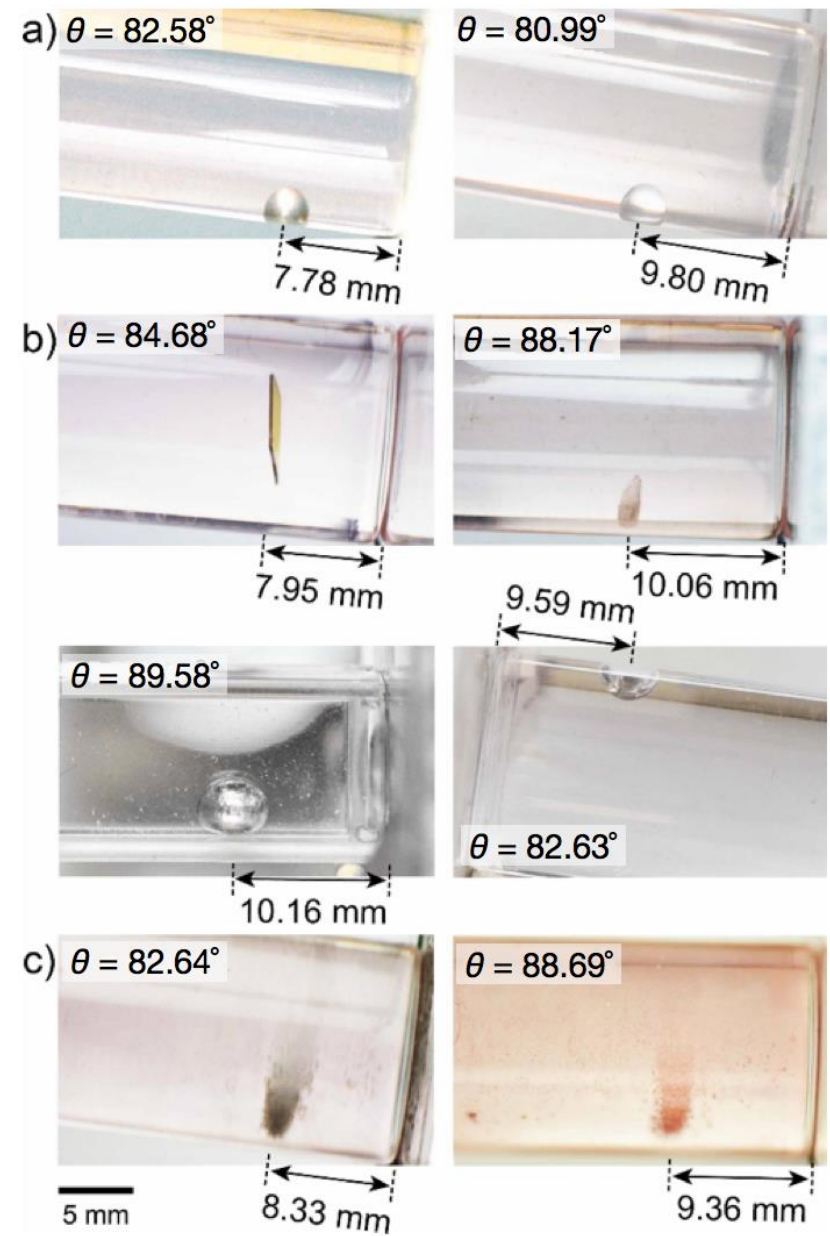

Figure 3. Measuring densities of different objects using the MagLev device. The angle of tilt $\theta$, and the distance $D_{\mathrm{z}}$ between the object and the surface of the bottom magnet used to calculate the densities are shown in the images. a) Measuring densities of spherical objects: (left) aluminum sphere; (right) glass sphere. b) Measuring densities of non-spherical objects: (top left) a flat piece of diamond; (top right) a piece of cerussite; (bottom left) a piece of iridium; (bottom right) an air bubble. Since the air bubble is less dense than the medium, it floats closer to the upper magnet than the lower magnet (distance as indicated). c) Measuring densities of powders: (left) aluminum powder; (right) copper powder. 
Table 1. Measurements of densities of spherical objects compared to values from literature.

\begin{tabular}{lccl}
\hline Material & $\begin{array}{l}\text { Known }^{\mathrm{a}} \\
\text { Density } \\
\left(\mathrm{g} / \mathrm{cm}^{3}\right)\end{array}$ & $\begin{array}{l}\text { Measured } \\
\text { Density } \\
\left(\mathrm{g} / \mathrm{cm}^{3}\right)\end{array}$ \\
\hline $\begin{array}{l}\text { High-Density Polyethylene } \\
\text { (HDPE) }\end{array}$ & 0.941 & $0.96 \pm 0.05$ \\
$\begin{array}{l}\text { Polytetrafluorethylene (Tef- } \\
\text { lon) }\end{array}$ & 2.21 & $2.2 \pm 0.04$ \\
Glass & $2.4-2.8$ & $2.4 \pm 0.04$ \\
Aluminum & 2.7 & $2.7 \pm 0.1$ \\
Silicon Nitride & 3.32 & $3.3 \pm 0.05$ \\
Aluminum Oxide & 3.88 & $3.9 \pm 0.06$ \\
Brass & 8.53 & $8.5 \pm 0.5$ \\
Lead & $11.2-11.3$ & 11 & \pm 0.6 \\
Mercury & 13.55 & 13 & \pm 0.9 \\
\hline${ }^{\mathrm{a} D a t a}$ were obtained from sources listed in the SI & \\
${ }^{b} \mathrm{n} \geq 7$ & \multicolumn{4}{l}{}
\end{tabular}

Table 2. Measurements of densities of non-spherical objects compared with values from literature.

\begin{tabular}{lcc}
\hline Material & $\begin{array}{l}\text { Known } \\
\text { Density } \\
\left(\mathrm{g} / \mathrm{cm}^{3}\right)\end{array}$ & $\begin{array}{l}\text { Measured } \\
\text { Density } \\
\left(\mathrm{g} / \mathrm{cm}^{3}\right)\end{array}$ \\
\hline Air & 0.001 & $0.0 \pm 0.04$ \\
Polytetrafluorethylene (Teflon) & 2.21 & $2.2 \pm 0.05$ \\
Silicon & 2.33 & $2.4 \pm 0.04$ \\
Diamond & 3.51 & $3.6 \pm 0.09$ \\
Aluminum $\mathrm{Oxide}^{\mathrm{b}}$ & 3.88 & $3.9 \pm 0.06$ \\
Stibnite $\left(\mathrm{Sb}_{2} \mathrm{~S}_{3}\right.$, mineral $)$ & $4.52-4.62$ & $4.5 \pm 0.1$ \\
Cerussite $\left(\mathrm{PbCO}_{3}\right.$, mineral $)$ & 6.55 & $6.5 \pm 0.2$ \\
Indium & 7.31 & $7.3 \pm 0.2$ \\
Copper & 8.96 & $9.0 \pm 0.6$ \\
Silver & 10.5 & $11 \pm 0.1$ \\
Lead & $11.2-11.3$ & $11 \pm 0.6$ \\
Gold & 19.3 & $20 \pm 1$ \\
Indium & 22.56 & $23 \pm 2$ \\
Osmium & 22.59 & $23 \pm 2$ \\
\hline
\end{tabular}

${ }^{\mathrm{a}}$ Data were obtained from sources listed in the SI

${ }^{b} \geq 7$

Measuring Densities of Multiple Objects Simultaneously. Increasing $\rho_{\text {range }}$ also enabled us to characterize multiple objects of widely differing density, simultaneously. To demonstrate this capability, we placed six different samples composed of i) alumimum, ii) poly(tetrafluoroethylene) (Teflon), iii) glass (manufacturer reported $\rho=1.8000 \mathrm{~g} / \mathrm{cm}^{3}$; American Density Materials, Inc.), iv) polyamide-imide (Torlon), v) polyamide (nylon 6/6), and vi) high-density polyethylene (HDPE), in a square cuvette in a MagLev device. Figure 4 shows how, after agitation, the objects distributed according density. The numeric values report the densities (averaged over seven trials, each at a different $\theta$ ) that we measured for each object by using Equation 7.

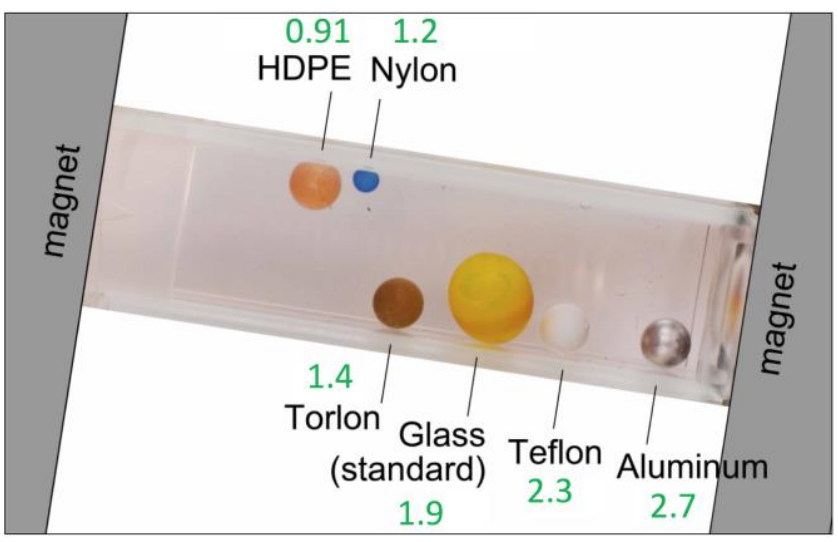

Figure 4. The simultaneous measurement of density of six spherical objects. The labeled values indicate the mean values $(n=7)$ of the measured densities $\left(\mathrm{g} / \mathrm{cm}^{3}\right)$.

Measuring Densities of Dense Powders. Because MagLev suspends samples in an aqueous medium without contact, it is also possible to use the increased $\Delta \rho_{\text {range }}$ to characterize a wide variety of powders composed of fine grains (tens to hundreds of micrometers) of dense materials (e.g., metallic powders). We tuned the viscosity of the medium with added dextran to attain a practical balance between rapid equilibriation (low viscosity enables the powder to settle to the bottom quickly) and ease of manipulation (high viscosity enables the powders to be "picked up" by rotation; see Experimental). Using this approach, we measured the densities of five different powders overall (gold, glass, aluminum, tin, and copper), with particle sizes ranging from -200 to 20 mesh $(74-841 \mu \mathrm{m})$. Figure $3 \mathrm{c}$ shows images of aluminum and copper powders sliding along the surface after we rotated the container. Table 3 reports the densities that we measured using this method compared to the known densities of these materials from literature. The densities measured by MagLev agree well with the values in the literature.

\section{CONCLUSION}

By tuning the buoyant force of gravity along the axis of measurement within a MagLev device, we have expanded the range of densities of non- or weakly-magnetic materials that can measured with this technique by $\sim 15 \mathrm{x}$ over previous ${ }^{2}$ efforts (from $\sim 0.8-2.3 \mathrm{~g} / \mathrm{cm}^{3}$ to $\sim 0.0-23 \mathrm{~g} / \mathrm{cm}^{3}$ ). This enhanced range enabled us to levitate and measure the density of objects with a wide variety of material compositions, including samples with either very low density (e.g., bubbles of air, foam) or very high density (e.g., osmium and iridium). By tuning the viscosity of the medium and periodically rotating the container before measurement, we were able to measure the density of objects with a wide variety of shapes and sizes, such as spherical particles (e.g., plastic beads), non-spherical samples (e.g., $\mathrm{Si}$ wafer, a flat sheet of artificial diamond), and even microscale powders.

The primary limitation to increasing the range of measurement emerges from the inherent trade-off between sensitivity and range: we must decrease the sensitivity to increase the range. It is difficult, therefore, to use this method to resolve small differences in density between samples that are both far from the density of the medium (e.g., metals) and physically large enough that they prevent each other, by hard contact, from reaching their equilibrium positions. This challenge, 
however, can be overcome by i) ensuring that the size of samples remains small (e.g., small particles or powders), ii) measuring the density and magnetic susceptibility of the medium with very high accuracy, and iii) using imaging equipment with high enough resolution to detect small differences or changes in position $(0.1-1 \mathrm{~mm})$. Alternatively, it may be possible to measure density with very high sensitivity (at the expense of range), by measuring along an axis that has a weak magnetic force (rather than along the axis of weak gravitational force). This approach, however, would only be effective for objects close in density of the medium, and not for the present case, where the densities of the samples are substantially different from that of the medium.

The methods presented in this paper expand the variety of possible applications available to MagLev. The ability to levitate, simultaneously, objects with a broad range of different densities may be useful in i) forensic science (e.g., for identifying the composition of miscellaneous objects or powders), ii) geology (e.g., for identifying small mineral grains), industrial manufacturing (e.g., for quality control of parts), iii) polymer science (e.g., for separating polymers based on crystallinity), iv) materials science (e.g., for identifying alloys of small particles), and v) resource-limited settings (e.g., for identifying and separating metals in developing countries). Our approach of tilting the MagLev device relative to the gravitational vector is operationally simple and can be implemented in the field with very simple equipment (e.g., by resting a MagLev device - two magnets and a container of fluidon its side, against a flat surface, such as a wooden plank, and measuring the angle with a digital level, such as a smartphone equipped with appropriate software).

\section{ASSOCIATED CONTENT}

\section{Supporting Information}

Supporting information includes supplementary materials and methods, literature values of densities, and supporting experimental design. The Supporting Information is available free of charge on the ACS Publications website.

\section{AUTHOR INFORMATION}

\section{Corresponding Author}

*Email: gwhitesides@gmwgroup.harvard.edu

\section{Author Contributions}

All authors have given approval to the final version of the manuscript. ${ }^{*}$ Authors contributed equally to this work.

\section{Funding Sources}

This work was funded by a grant from the Department of Energy, Division of Materials Science and Engineering under award ER45852. H-D.Y. acknowledges the Agency for Science, Technology and Research (A*STAR) International Fellowship from the
Singapore Government.

\section{ACKNOWLEDGEMENTS}

We thank Prof. Marko Loncar (Harvard School of Engineering and Applied Sciences) for providing the diamond used in this study and Dr. Ashok. A. Kumar for useful discussions.

\section{REFERENCES}

(1) Mirica, K. A.; Phillips, S. T.; Shevkoplyas, S. S.; Whitesides, G. M. J. Am. Chem. Soc. 2008, 130, 17678-17680.

(2) Mirica, K. A.; Shevkoplyas, S. S.; Phillips, S. T.; Gupta, M.; Whitesides, G. M. J. Am. Chem. Soc. 2009, 131, 10049-10058.

(3) Mirica, K. A.; Phillips, S. T.; Mace, C. R.; Whitesides, G. M. J. Agric. Food Chem. 2010, 58, 6565-6569.

(4) Lockett, M. R.; Mirica, K. A.; Mace, C. R.; Blackledge, R. D.; Whitesides, G. M. J. Forensic Sci. 2013, 58, 40-45.

(5) Tasoglu, S.; Kavaz, D.; Gurkan, U. A.; Guven, S.; Chen, P.; Zheng, R.; Demirci, U. Adv. Mater. 2013, 25, 1137-43-1081.

(6) Tasoglu, S.; Yu, C. H.; Gungordu, H. I.; Guven, S.; Vural, T.; Demirci, U. Nature Comm. 2014, 5, 4702-4711.

(7) Atkinson, M. B. J.; Bwambok, D. K.; Chen, J.; Chopade, P. D.; Thuo, M. M.; Mace, C. R.; Mirica, K. A.; Kumar, A. A.; Myerson, A. S.; Whitesides, G. M. Angew. Chem. 2013, 52, 1020810211.

(8) Eren, H. In The Measurement, Instrumentation and Sensors Handbook on CD-ROM; CRC Press, 2014.

(9) Ilievski, F.; Mirica, K. A.; Ellerbee, A. K.; Whitesides, G. M. Soft Matter 2011, 7, 9113.

(10) Mirica, K. A.; Ilievski, F.; Ellerbee, A. K.; Shevkoplyas, S. S.; Whitesides, G. M. Adv. Mater. 2011, 23, 4134-4140.

(11) Subramaniam, A. B.; Yang, D.; Yu, H.-D.; Nemiroski, A.; Tricard, S.; Ellerbee, A. K.; Soh, S.; Whitesides, G. M. Proc. Natl. Acad. Sci. U.S.A. 2014, 111, 12980-12985.

(12) Hennek, J. W.; Nemiroski, A.; Subramaniam, A. B.; Bwambok, D. K.; Yang, D.; Harburg, D. V.; Tricard, S.; Ellerbee, A. K.; Whitesides, G. M. Adv. Mater. 2015, 27, 1587-1592.

(13) Shapiro, N. D.; Soh, S.; Mirica, K. A.; Whitesides, G. M. Anal. Chem. 2012, 84, 6166-6172.

(14) Shapiro, N. D.; Mirica, K. A.; Soh, S.; Phillips, S. T.; Taran, O.; Mace, C. R.; Shevkoplyas, S. S.; Whitesides, G. M. J. Am. Chem. Soc. 2012, 134, 5637-5646.

(15) Kumar, A. A.; Patton, M. R.; Hennek, J. W.; Lee, S. Y. R.; D’Alesio-Spina, G.; Yang, X.; Kanter, J.; Shevkoplyas, S. S.; Brugnara, C.; Whitesides, G. M. Proc. Natl. Acad. Sci. U.S.A 2014, 111, 14864-14869.

(16) Subramaniam, A. B.; Gonidec, M.; Shapiro, N. D.; Kresse, K. M.; Whitesides, G. M. Lab Chip 2015, 15, 1009-1022.

(17) Durmus, N. G.; Tekin, H. C.; Guven, S.; Sridhar, K.; Arslan Yildiz, A.; Calibasi, G.; Ghiran, I.; Davis, R. W.; Steinmetz, L. M.; Demirci, U. Proc. Natl. Acad. Sci. U.S.A. 2015, 112, E3661E3668.

(18) Xu, X.; Li, H.; Zhang, Z.; Qi, X. J. Appl. Polym. Sci. 2009, 111, $1523-1529$ 
TOC Image

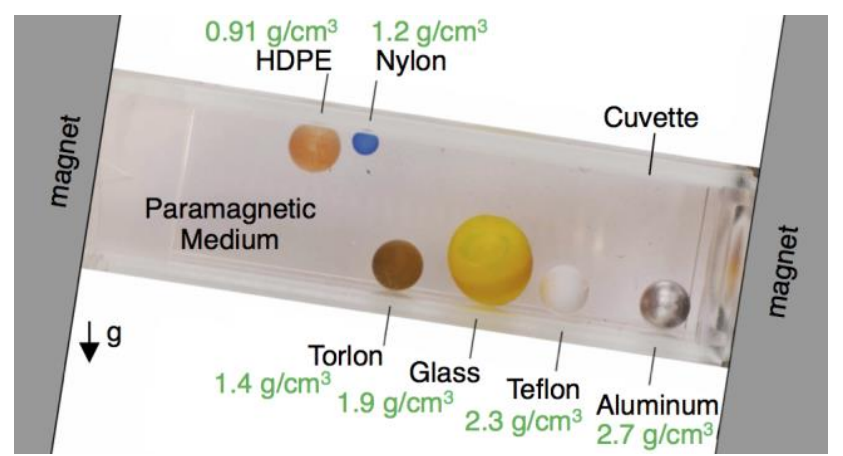

